\title{
World Congress on Osteoporosis, Osteoarthritis and Musculoskeletal Diseases (WCO-IOF-ESCEO 2020): Special Lecture Abstract
}

\author{
(C) International Osteoporosis Foundation and National Osteoporosis Foundation 2020
}

\section{SL1}

ESCEO-IOF ALGORITHM FOR THE MANAGEMENT OF PATIENTS AT LOW/HIGH/VERY HIGH RISK OF FRACTURE J. A. Kanis $^{1,2}$, N. C. Harvey ${ }^{3,4}$, E. V. Mccloskey ${ }^{5}$, O. Bruyère ${ }^{6}$, N.

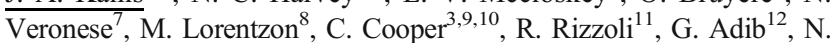
Al-Daghri $^{13}$, C. Campusano ${ }^{14}$, M. Chandran ${ }^{15}$, B. Dawson-Hughes ${ }^{16}, \mathrm{M}$. K. Javaid ${ }^{9}$, F. Jiwa ${ }^{17}$, H. Johansson ${ }^{1,2}$, J. K. Lee ${ }^{18}$, E. Liu ${ }^{2}$, O. D. Messina $^{19}$, O. Mkinsi ${ }^{20}$, D. Pinto ${ }^{21}$, D. Prieto-Alhambra ${ }^{9}$, K. Saag ${ }^{22}$, W. Xia ${ }^{23}$, L. Zakraoui ${ }^{24}$, J.-Y. Reginster ${ }^{13,25,26}$

${ }^{1}$ Centre for Metabolic Bone Diseases, University of Sheffield Medical School, Sheffield, United Kingdom, ${ }^{2}$ Mary McKillop Health Institute, Australian Catholic University, Melbourne, Australia, ${ }^{3} \mathrm{MRC}$ Lifecourse Epidemiology Unit, University of Southampton, Southampton, United Kingdom, ${ }^{4}$ NIHR Southampton Biomedical Research Centre, University of Southampton and University Hospital Southampton NHS Foundation Trust, Southampton, United Kingdom, ${ }^{5}$ Department of Oncology \& Metabolism, University of Sheffield, Sheffield, United Kingdom, ${ }^{6}$ Public Health, Epidemiology \& Health Economics, University of Liège, Liège, Belgium, ${ }^{7}$ National Research Council, Neuroscience Institute, Aging Branch, Padova, Italy, ${ }^{8}$ Geriatric Medicine, Sahlgrenska University Hospital, Mölndal, Sweden, ${ }^{9}$ Nuffield Department of Orthopaedics, Rheumatology and Musculoskeletal Sciences, University of Oxford, Oxford, United Kingdom, ${ }^{10}$ Institute of Musculoskeletal Sciences, University of Oxford, Oxford, United Kingdom, ${ }^{11}$ Division of Bone Diseases, Geneva University Hospitals and Faculty of Medicine, Geneva, Switzerland, ${ }^{12}$ Osteoporosis Center, Italien Hospital, Damascus-Syria, Damascus, Syria, ${ }^{13}$ Chair for Biomarkers of Chronic Diseases, Biochemistry Department, College of Science, King Saud University, Riyadh, Saudi Arabia, ${ }^{14}$ Clinica universidad de los andes, Santiago, Chile, ${ }^{15}$ Osteoporosis and Bone Metabolism Unit, Singapore General Hospital, Singapore, Singapore, ${ }^{16}$ Jean Mayer USDA Human Nutrition Research Center on Aging at Tufts University, Boston, United States, ${ }^{17}$ Osteoporosis Canada, Toronto, Canada, ${ }^{18}$ Beacon Hospital, Petaling Jaya, Malaysia, ${ }^{19}$ Rheumatology Service, Cosme Argerich Hospital and IRO ClinicalResearch Center, Buenos Aires, Argentina, ${ }^{20} \mathrm{CHU}$ Ibn Rochd, Casablanca, Morocco, ${ }^{21}$ Marquette University/Department of Physical Therapy, Milwaukee, United States, ${ }^{22}$ University of Alabama, Birmingham, United States, ${ }^{23}$ Peking Union Medical College Hospital (East), Beijing, China, ${ }^{24}$ Hospital Mongi Slim at La Marsa, Tunis, Tunisia, ${ }^{25}$ Department of Public Health, Epidemiology and Health
Economics, University of Liège, Liège, Belgium, ${ }^{26}$ WHO Collaborating Center for Public Health Aspects of Musculoskeletal Health and Aging, Liège, Belgium

In 2019 the International Osteoporosis Foundation (IOF) and the European Society for Clinical and Economic Evaluation of Osteoporosis and Osteoarthritis (ESCEO) published updated guidance for the diagnosis and management of postmenopausal osteoporosis. The algorithm supplements this guidance to recognise that the risk of a subsequent osteoporotic fracture is particularly acute immediately after an index fracture and wanes progressively with time. Additionally, new anabolic agents with more rapid and greater fracture risk reduction compared to antiresorptive treatments have been developed. These have the potential to revolutionise treatment strategies, particularly in individuals at very high fracture risk. These considerations argue for the identification of individuals at very high risk of fracture.

The algorithm follows the guidance of the IOF and ESCEO in the use of age-dependent intervention thresholds with the use of FRAX. In addition to the categories of low and high risk espoused in the current IOF-ESCEO guideline, very high risk can be identified as a fracture probability that exceeds the current intervention threshold by $20 \%$. In women age 50 years or more from the UK, $64.8 \%$ would be categorised at low risk, $19.7 \%$ at high risk and $15.6 \%$ at very high risk. A FRAX adjustment is provided to take account that the probability of second fracture is particularly high in the first 2 years after a clinical vertebral fracture. The $10-$ year probability of a major osteoporotic fracture is multiplied by 1.04 to 2.47 , depending on age. FRAX adjustments are still needed men and for index fractures other than spine fracture.

The rationale for the more refined characterisation of risk is to direct appropriate interventions. Thus, initial treatment recommendations for women at high risk might most usually start with an inhibitor of bone resorption. In contrast, women at very high risk might be more suitably treated with an anabolic treatment followed thereafter by an inhibitor of bone resorption. Such regimens save more fractures than inhibitors of bone resorption followed by anabolic agents.

Publisher's note Springer Nature remains neutral with regard to jurisdictional claims in published maps and institutional affiliations. 